\title{
DT56a in treatment of climacteric syndrome in the Central European population sample
}

\author{
Fait $\mathrm{T}^{1}$, Borovsky $\mathrm{M}^{2}$ \\ Department of Obstetrics and Gynaecology, 2nd Faculty of Medicine, Charles University Prague \\ at Faculty Hospital Motol, Prague, Czech Republic. tfait@seznam.cz
}

\section{ABSTRACT}

OBJECTIVES: The aim of this study was to assess the possibility of using the DT56a for the therapy of acute climacteric syndrome in women in Slovakia and the Czech Republic.

DESIGN: Prospective interventional unblinded study.

RESULTS: A sample of 453 women with symptoms of acute climacteric syndrome took $644 \mathrm{mg}$ of soybeans' phyotestrogenes DT56a in the period of 4 weeks. In course of the therapy, the total number of hot flushes decreased by $48 \%$, and the intensity decreased by $35 \%(p<0.01)$. In $85 \%$ of women, the quantity or intensity of hot flushes decreased. Sleep quality increased in $65 \%$ of women, headaches improved or significantly improved in $51 \%$ of women, muscle aches and joint pains decreased by $40 \%$. Life quality improved in $72 \%$ of women.

CONCLUSIONS: DT56a is a possible alternative for the treatment of acute climacteric syndrome. In the Central European population, the efficacy of a daily dose of $644 \mathrm{mg}$ corresponds with the effects observed in the Mediterranean population (Tab. 2, Fig. 3, Ref. 21). Text in PDF www.elis.sk

KEY WORDS: DT56a, climacteric syndrome, menopause, non-hormonal treatment.

\section{Introduction}

Selective estrogenic receptor modulators (SERM) are defined as substances, which have different agonist/antagonist effects in various tissues. The substance DT56a has such properties. It alleviates symptoms of acute climacteric syndrome and increases bone mineral density without an influence on blood steroids levels, endometrial thickness and breast tissue $(1,2)$.

From the break of last centuries women are commonly afraid of hormone therapy and prefer "natural" solution for their symptoms of menopause (3) especially after the publication of Women Health Initiative Study results in daily press (4).

Recently published data assessed the efficacy of DT56a on a sample of Greek (5) and Spanish (6) population. The goal of our study was to assess the efficacy of this treatment in the Central European population. In order to achieve comparability, we opted for the same design as the Spanish POMMSIS (Post Marketing Menopausal Symptoms International Survey) and designated the study as POMMSIS CZ-SK.

${ }^{1}$ Department of Obstetrics and Gynaecology, 2nd Faculty of Medicine, Charles University Prague at Faculty Hospital Motol, Prague, Czech Republic, and ${ }^{2} 1$ st Department of Gyneacology and Obstetrics, Comenius University Bratislava, St. Cyril a Method's Hospital, Bratislava, Slovakia Address for correspondence: T. Fait, MD, PhD, Faculty Hospital Motol, V Uvalu 84, CZ-150 00 Prague 5, Czech Republic.

Phone: +420 603910473

\section{Patients and methods}

In the Czech Republic and Slovakia, 52 gynaecological centres were selected in both urban and rural areas. In total, 459 women were included in the study, 453 concluded the study. Among 6 women which did not finish study, there were 2 with worsening of hot flushes, 1 with headache, 1 with nausea and 2 missing without information.

Inclusion criteria: Climacteric syndrome with at least 1 hot flush a day, preferring non-hormonal treatment.

Exclusion criteria: A criterion for elimination was a hormonal treatment in the previous 3 months.

The patients took 1 pill of Femarelle ${ }^{\circledR}$ (Se-cure Pharmaceuticals, Israel) twice each day. Each capsule contains $322 \mathrm{mg}$ fer-

Tab. 1. Soy isoflavones in DT56a.

\begin{tabular}{lc}
\hline Type of isoflavones & Concentration $(\mathrm{mg} / \mathrm{g})$ \\
\hline Genistin & 0.243 \\
Genistein & 0.095 \\
Glycitin & 0.044 \\
Glycitein & 0.013 \\
Daidzin & 0.184 \\
Daidzein & 0.086 \\
isoflavones total & 1.02 \\
6'-O-acetyldaidzin & $<0.01$ \\
6'-O-acetylgeniston & 0.034 \\
6'-O-acetylglycitin & 0.028 \\
6'-O-malonyldaidzin & $<0.01$ \\
6'-O-malonylgenistin & 0.289 \\
6'-O-malonylglycitin & $<0.01$ \\
\hline
\end{tabular}


Tab. 2. Syndrome intensity prior to treatment.

\begin{tabular}{lcccc}
\hline$\%$ & $\begin{array}{c}\text { Hot flush } \\
\text { (n 450) }\end{array}$ & $\begin{array}{c}\text { Night sweating } \\
\text { (n 444) }\end{array}$ & $\begin{array}{c}\text { Headaches } \\
\text { (n 357) }\end{array}$ & $\begin{array}{c}\text { Joint pains and muscle aches } \\
\text { (n 387) }\end{array}$ \\
\hline insignificant & 1 & 6 & 41 & 24 \\
low & 7 & 14 & 26 & 25 \\
medium & 38 & 34 & 21 & 29 \\
common & 39 & 30 & 9 & 16 \\
very common & 15 & 16 & 3 & 6 \\
average \pm SD & $3.59 \pm 0.86$ & $3.34 \pm 1.086$ & $2.05 \pm 1.094$ & $2.55 \pm 1.18$ \\
\hline
\end{tabular}

a surgical menopause after adnexectomy, with the average age of $47 \pm 4.9$ years, in $74 \%$ it was a natural menopause starting on $50 \pm 3.2$ years.

The mean quantity of hot flushes before the treatment was $7 \pm 6.9$, out of which $37 \%$ women described no more than 4 hot flushes daily, $63 \% 6$ and more (Fig. 1, Tab. $2)$. The average intensity of hot flushes was $3.59 \pm 0.86$.

mented soy derivate DT56a and $108 \mathrm{mg}$ flaxseed. Each capsule contains 2,5 ng of coumestrol, $1488 \mathrm{ng}$ of equol and $1974 \mathrm{ng}$ of genistein. Due to the fermentation, there are 12 phytoestrogens in DT56a (Tab. 1).

In the beginning of the study, and after 5 weeks, the women and physicians filled a questionnaire regarding the quantity and intensity of hot flushes, sleep quality, headaches, muscle aches and joint pains.

On daily basis, the patients filled in questionnaires regarding the quantity and intensity of hot flushes. The first week was for reference, during the following 4 weeks, they were taking the assessed product. The statistical software IBM SPSS Statistic Base version 22.0 was used to analyse the data. The data are expressed as the absolute numbers and percentages or as the means and standard deviations. Normally distributed continuous data were compared to Student's t-test or an analysis of variance (ANOVA). When data distributions departed from normality, the Mann-Whitney U-test or the Kruskal-Wallis test was used. The $\mathrm{X}^{2}$ text was used to compare categorical variable data. A value of $p<0.05$ was considered statistically significant for all statistical tests performed.

\section{Results}

The average age of the women was $46 \pm 5.6$ years, weight 72 $\pm 13.8 \mathrm{~kg}$, height $165 \pm 6.01 \mathrm{~cm}$. Smokers comprised $17 \%$ of the sample. $66 \%$ was post-menopausal, $34 \%$ was in perimenopause. The mean menopausal age was 49 years, in $26 \%$ women it was

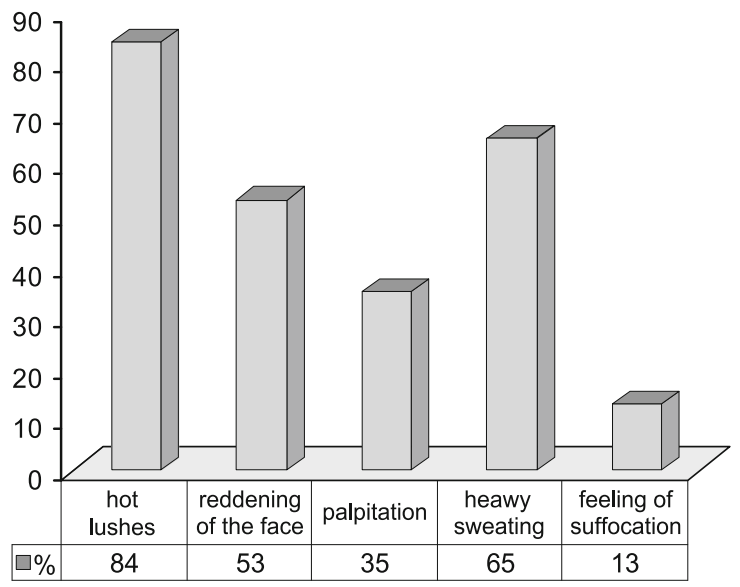

Fig. 1. Typical daily symptoms (n 453).
A decreased sleep quality was reported by $52 \%$ of women. Significant muscle aches and joint pains were reported by $51 \%$ of respondents.

In the course of the 4 weeks treatment, the total number of daily hot flushes decreased by $48 \%$ from $7 \pm 6.92$ to $3.62 \pm 4.62$. The reduction on weekly basis was significant $(\mathrm{p}<0.01)$ (Fig. 2). Similarly, the intensity of hot flushes declined $(\mathrm{p}<0.01)$ on weekly basis by $9 \%, 13 \%, 11 \%$, and $8 \%$, which is by $35 \%$ after the month. There was a drop in quantity or intensity of hot flushes in $85 \%$. Sleep quality improved in $65 \%$ women, headaches improved or significantly improved in $51 \%$, and muscle aches and joint pains in $40 \%$ women. Life quality increased in $72 \%$.

Also, in the subgroup of women with 7 or more flushes daily (n $160)$, there was a significant $(\mathrm{p}<0.01)$ diminution in the quantity, from 10.5 to 6 , as well as in the intensity of hot flushes from 3.4 to 2.5 . The intensity decreased by $16 \%$ after 2 weeks, by $34 \%$ in 4 weeks. The quantity of hot flushes fell after a 4 weeks treatment by $43 \%$. The drop in quantity or intensity of hot flushes was $86 \%$ in the subgroup of a strong climacteric syndrome.

\section{Discussion}

The studies showed certain differences in the symptoms of the climacteric syndrome in various categories, impacted by a number of variables $(7,8)$, for example by climate, diet, or lifestyle. These factors are entirely different in Central Europe and the Mediterranean.

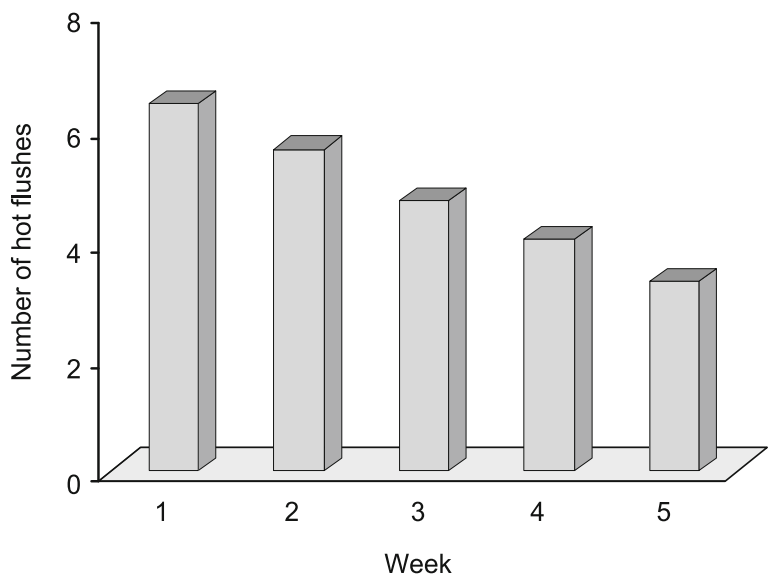

Fig. 2. Decrease in quantity of hot flushes during the treatment (weekly average). 


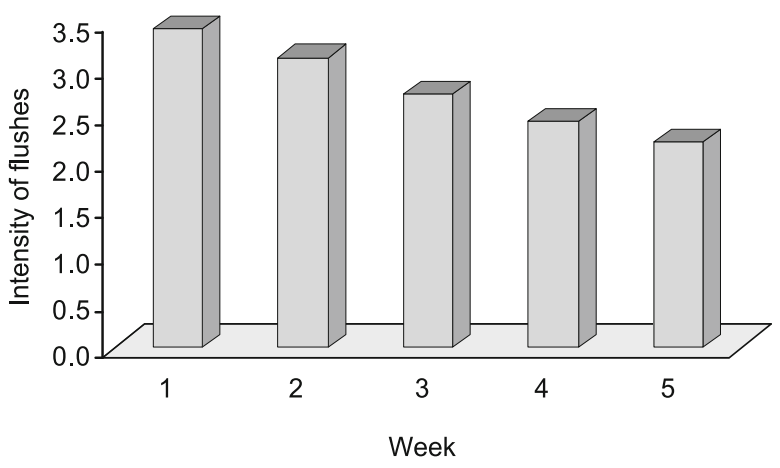

Fig. 3. Decrease in intensity of hot flushes.

Our study is the first for using DT56a for therapy of acute climacteric syndrome in the Central Europe population. The limitation of study is nonblinded and no placebo control design.

\section{Preclinical studies}

The identical effect of DT56a and estradiol on the vessel wall was proven on a rat model by means of creatine kinase activity (9). Also, the impact on opioid systems, neurosteroid allopregnanolone and gamma-aminobutyric acid is comparable for estrogens and DT56a (10).

DT56a stimulates creatine kinase and DNA synthesis in the tissue culture of female osteoblasts in pre-menopause as well as post-menopause with maximal effect with the concentration of $100 \mathrm{ng} / \mathrm{ml}$. This DT56a effect could be blocked by raloxifene. On the contrary, DT56a entirely blocks the effects of estradiol, if administered simultaneously. Proportionately to the dosage, DT56a increases the intercellular level of calcium with the highest effect at the level of $10 \mathrm{ng} / \mathrm{ml}$ (11). The estradiol effect on female osteoblasts decreases in hyperglycaemic condition. DT56a works independently on the glucose levels. It could be important in patients suffering from diabetes (12).

Application of DT56a or estradiol was observed in two groups of rats in the duration of 8 weeks. The neutered rats taking the placebo lost $31 \%$ of trabecular $(\mathrm{p}<0.01)$ and $21 \%$ of cortical bone $(\mathrm{p}<0.001)$. Administration of DT56a led to significant growth of cortical bone thickness, $32 \%$ in neutered, and $38 \%$ in non-neutered mice. Creatine kinase activity increased after administration of estradiol in the pineal gland and diaphyseal femur, as well as in DT56a, which however, unlike as in the case of estradiol, did not increased this activity in the tissue of uterus (13).

Another study on the model of neutered rats confirmed the DT56a function corresponding with SERM. While a single hypodermic administration of a high dosage of DT56a indicated the same creatine kinase activity in bone tissue as well as in the uterus tissue as estradiol, a repeated oral administration of DT56a induced the same activity in the bone tissue but not in the uterus tissue. In both observed cases, the estradiol and DT56a effect was blocked by tamoxifen (14).

In neutered rats, osteoformation decreases rapidly and the percentage of fat cells in the bone fibre increases. These two pro- cesses have an inverse character. Estradiol, raloxifene and DT56a lead to almost complete disappearance of fat cells, daidzein decreased their representation by $80 \%$, genistein and biochanin A had no impact. Vitamin $\mathrm{K}$ analogues did not impact the content of the cells, but in combination with estradiol, led to their complete disappearance (15).

In the study specifically aimed at this issue, neutered rats were orally administered with 3.4 or $10.2 \mathrm{mg} / \mathrm{kg}$ of DT56a in comparison with 31 or $100 \mu \mathrm{g} / \mathrm{kg}$ of conjugated equol estrogens (CEE) for the period of 14 days. DT56a, unlike CEE, did not lead to any measurable increased in estrogen activity in the rats' genitals (16).

In the human breast carcinoma cells culture, DT56a in dosage corresponding to $644 \mathrm{mg}$ daily did not impact growth (17). Genistein could have positive effects also on other tissues (18).

\section{Clinical studies}

At first, the study using DT56a for osteoporosis prevention was published. In the group of 98 healthy postmenopausal women, the impact of $644 \mathrm{mg}$ and $322 \mathrm{mg}$ DT56a on bone density during a one-year long administration was studied. Bone mineral density (BMD) at the dose of $644 \mathrm{mg}$ daily increased in the lumbar area by $3.6 \%$ and in the femoral neck by $2 \%$, in case of lower dosage it decreased in both locations by $0.6 \%$, the difference in the lumbar area was significant $(\mathrm{p}=0.037)$. The impact on hormonal levels, endometrial thickness or other side effects were not observed (19).

Subsequently, the same scientific unit published an observing of impact on acute climacteric syndrome. The group of 80 symptomatic postmenopausal women was randomised for $644 \mathrm{mg}$ or 322 mg DT56a daily. Check-ups were performed every three months for a period of one year. In both groups, there was a significant $(p<0.01)$ decrease of Kupperman Index after 12 weeks, which prevailed even after 12 months. $78 \%$ of respondents on lower dose and $76 \%$ on higher dose reported alleviation of vasomotor symptoms. No changes in TSH levels, steroid hormones or endometrial thickness were observed. The effect on the acute climacteric syndrome is the same for both doses of DT56a (20).

The Greek prospective study was similar in its scope, where 89 postmenopausal women with acute climacteric syndrome were randomised for $644 \mathrm{mg}$ of DT56a daily (27 women), low dosage hormonal substitution therapy $1 \mathrm{mg}$ estradiol $+0.5 \mathrm{mg}$ norethisterone acetate (26 women) and no therapy (36 women). DT56a and hormonal therapy was connected to a significant $(\mathrm{p}<0.001)$ decrease of Kupperman Index -3.98 as opposed to -5.06$)$ as compared to the no therapy group $(+1.76)$. The women who were not treated, displayed a significant decrease in bone mineral density (BMD) in the lumbar spine according to the T-score formula (from -0.6 to $0.85, \mathrm{p}=0.001$ ), which did not change in the treated group. There was no change in the bone density of the femoral neck, mammographic findings or endometrial thickness. There was also no change in the lipid profile (5).

So far, the most extensive study was published in 2015. The group of 631 peri-menopausal and menopausal women with acute climacteric syndrome was administered DT56a for the period of 4 weeks. After 2 and 4 weeks, $80.7 \%$ participants reported a significant decrease in the quantity of hot flushes $(p<0.01)$. Intensity 


\section{1-304}

of hot flushes decreased in $38 \%$ of women, who had more than 7 hot flushes a day, before joining the study (6).

The impact of administering $644 \mathrm{mg}$ DT56a daily for 8 weeks on the platelet function was observed by means of PFA-100 (Platelet Function Analyser - 100). In the aggregate of symptomatic post-menopausal women, there was a group of 25 healthy women and 7 women with a shortened coagulation time $(4 \mathrm{x}$ heterozygote Leiden, 1 heterozygote for prothrombin mutation, 1 protein $\mathrm{S}$ deficit, 1 increase in antiphospholipid antibodies). A positive impact on the acute climacteric syndrome was recorded in all women. The coagulable risk increased in none (21).

\section{Conclusion}

The DT56a made by an extraction of fermented genetically not modified soya beans contains a mix of phytoestrogens which act as an ideal SERM in both clinical and preclinical studies. In the daily dose of $644 \mathrm{mg}$, it has a comparable impact on acute climacteric syndrome, as compared to hormone replacement therapy.

At the same time, it has neutral and - in the presence of estrogens - antiestrogenic impact on the breast tissue and uterus. It does not affect the estrogen levels, gonadoliberins, thyroid hormones, lipid profile, liver function or coagulation of blood.

The studies show certain differences in the symptoms of the climacteric syndrome in various categories, impacted by a number of variables for example by climate, diet, or lifestyle. These factors are entirely different in Central Europe and the Mediterranean.

Nonetheless, our study has proven a comparable efficiency of the DT56a extract in a sample of Central European population, comparable to the large study conducted in Spain.

\section{References}

1. Sánchez-Borrego R, Navarro MC, Llaneza P et al. Efficacy and safety of a phyto-SERM as an alternative to hormone therapy. Climacteric 2014; $3: 1-8$

2. Somjen D, Katzburg S, Knoll E et al. DT56a (Femarelle): a Natural Selective Estrogen Receptor Modulator (SERM). J Steroid Biochem Mol Biol 2007; 104: 252-258.

3. De Franciscis P, Colacurci N, Riemma G et al. A nutraceutical approach to menopausal complaints. Medicina (Kaunas) 2019; 55 (9): 544.

4. Fait T. Menopause hormone therapy: latest developments and clinical practice. Drugs Context 2019; 8: 212551.

5. Labos G, Trakakis E, Pliatsika P et al. Efficacy and safety of DT56a compared to hormone therapy in Greek postmenopausal women. J Endocrinol Invest 2013; 36: 521-526.

6. Sánchez-Borrego R, Mendoza N, Llaneza P. A prospective study of DT56a (Femarelle $\left.{ }^{\circledR}\right)$ for the treatment of menopause symptoms. Climacteric $2015 ; 10: 1-4$.
7. Freeman EW, Sheriff K. Prevalence of hot flushes and night sweats around the world: a systematic review. Climacteric 2007; 10 (3): 197-214.

8. Santoro N, Sutton-Tyrrell K. The SWAN song: Study of Women's Health Across the Nation's recurring themes. Obstet Gynecol Clin North Am 2011; 38 (3): 417-423.

9. Somjen D, Yoles I. DT56a stimulans creatine kinase specific activity in vascular tissues of rats. J Endocrinol Invest 2003; 26: 966-971.

10. Pluchino NP, Merlini S, Cubeddu A et al. Brain-region responsiveness to DT56a (Femarelle) administration allopregnanolone and opioid content in ovaryectomized rats. Menopause 2009; 16 (5): 1037-1043.

11. Somjen D, Katzburg S. Lieberherr M et al. DT56a Stimulates Gender-Specific Human Cultured Bone Cells In-Vitro. J Steroid Biochem Mol Biol 2006; 98 (1): 90-96.

12. Somjen D et al. DT56a (Femarelle); contrary to estradiol-17 $\beta$; is effective in human deriveted female osteoblasts in hyperglycemic condition. J Steroid Biochem Mol Biol 2011; 123: 25-29.

13. Somjen D, Katzburg S, Livne E, Yoles I. DT56a (Femarelle) Stimulates Bone Formation in Female Rats. Brit J Obstet Gynecol 2005; 112 (7): 981-985.

14. Somjen D, Yoles I. DT56a (Tofupill/Femarelle) selectively stimulates creatine kinase specific activity in skeletal tissues of rats but not in the uterus. J Steroid Biochem Mol Biol 2003; 86: 93-98.

15. Somjen D, Katzburg S, Kohen $\mathbf{F}$ et al. The effects of native and synthetic estrogenic compounds as well as vitamin D less-calcemic analogs on adipocytes content in rat bone marrow. J Endocrinol Invest 2011; 34: $106-110$

16. Oropeza MV, Orozco S, Ponce H, Campos MG. Tofupill lack peripheral estrogen-like actions in the rat reproductive tract. Reproduct Toxicol 2005; 20: 261-266.

17. Yoles I, Lilling G. Pharmacological Doses of the Natural phyto-SERM DT56a (Femarelle) Have no Effect on MCF-7 Breast Cancer Cell-Line. Eur J Obstetrics Gynecol Reprod Biol 2006; 130 (1): 140-141.

18. Akinci O, Durgun V, Kepil $\mathbf{N}$ et al. The role of genistein in experimental hepatic ischemia-reperfusion model in rats. Bratisl Med J 2019; 120 (8): 558-562.

19. Yoles I, Yogev Y, Frenkel Y et al. Tofupill/Femarelle (DT56a) - a new phyto-selective estrogen receptor modulator-like substance for the treatment of postmenopausal bone loss. Menopause 2003; 10 (6): 522-525.

20. Yoles I, Yogev Y, Frenkel Y et al. Efficacy and Safety of Standard versus Low Dose of Femarelle (DT56a) for the Treatment of Menopausal Symptoms. Clin Exper Obstet Gynecol 2004; 31 (2): 123-126.

21. Nachtigall MJ, Jessel RH, Flaumenhaft $R$ et al. The selective estrogen receptor modulator DT56a (Femarelle) does not affect platelet reactivity in normal or thrombophilic postmenopausal women. Menopause 2011; 18 (3): 285-288.

Received November 22, 2020. Accepted December 4, 2020. 